\title{
Introduction to the HICSS-50 Organizational Issues of Business Intelligence, Business Analytics and Big Data Minitrack
}

\author{
Olivera Marjanovic \\ University of Sydney \\ Australia \\ olivera.marjanovic@, \\ sydney.edu.au
}

\author{
Barbara Dinter \\ Chemnitz University of \\ Technology, Germany \\ barbara.dinter@wirtschaft.tu- \\ chemnitz.de
}

\author{
Thilini Ariyachandra \\ Xavier University \\ USA \\ ariyachandrat@xavier.edu
}

Recent developments and the evolution of data and technologies in the business intelligence (BI) and business analytics (BA) space have given rise to new opportunities, innovation and challenges for individuals, organizations and society. The Internet of things has begun to create more demand for real time analytics and visualization. The emergence of major vendors (such as Amazon and Microsoft) in the cloud services arena is making cloud based BI a reality for both small and medium businesses as well as large corporations. The more prevalent use and adoption of predictive analytics and data science is heightening BI and analytics skill shortages experienced by companies.

Meanwhile, there is an ongoing major shift in the industry of organizations leveraging pervasive, selfservice analytics. The push for self-service analytics that include a portfolio of analysis tools, algorithms and visualizations has enabled agile decision making through effective business insights. In addition, the growth in this space has led to societal implications, both positive and negative.

The evolution and innovation experienced has created a state of excitement and flux in the BI, BA and big data space. Starting from a rich historic advancement that spanned from the development of Management Information Systems, Decision Support Systems, Executive Information Systems, interactive online analysis (OLAP), data mining, dashboards, predictive analytics, to more recently big data, Internet of things, machine learning and cognitive analytics, this space continues to present a wide variety of research questions and challenges for researchers to explore.

This year's minitrack includes the following four research papers:

- The opening paper by Marjanovic and Dinter is inspired by the $50^{\text {th }}$ anniversary of the Hawaii International Conferences on Systems Sciences (HICSS). The authors report on the 27-year history of interesting and relevant research included in this longest-running minitrack on BI, BA and big data at HICSS. The paper offers an overview of the key research themes and their progress over time obtained through a semantic text mining of all research publications included in this minitrack since 1990.

- Someh, Wixom, Davern and Shanks investigate possible enablers and mechanisms of synergy between BA and other organizational systems. Using a case study of a large IT firm the authors identify a set of organizational practices that underlie the emergence of higher-order BA-enabled organizational systems.

- Schuritz, Seebacher and Dorner provide an overview of possible revenue models for data-driven services. Through a qualitative analysis of 100 startups, the authors identify different revenue models such as subscription, gain sharing and multi-sided revenue models.

- The final paper by Yang, Pinsonneault and Hsieh explores how user engagement affects their intention to explore BI systems. Their lab experiment suggests that the cognitive fit and the regulatory compatibility influence users' engagement experience, which in turn affect their intention to explore BI systems.

We hope that you will find this year's selection of papers interesting and relevant. In the spirit of the $50^{\text {th }}$ Anniversary of HICSS, we also hope that you will continue to support this longest running minitrack on BI, BA and big data at HICSS for many years to come. 